\title{
Gemini South multi-conjugate adaptive optics (GeMS) laser guide star facility on-sky performance results
}

\author{
Céline d'Orgeville*a, ${ }^{\text {b }}$, Sarah Diggs ${ }^{\mathrm{b}}$, Vincent Fesquet ${ }^{\mathrm{b}}$, Benoit Neichel ${ }^{\mathrm{b}}$, William Rambold ${ }^{\mathrm{b}}$, \\ François Rigaut $^{\mathrm{a}, \mathrm{b}}$, Andrew Serio ${ }^{\mathrm{b}}$, Claudio Araya ${ }^{\mathrm{b}}$, Gustavo Arriagada ${ }^{\mathrm{b}}$, Rodrigo Balladares ${ }^{\mathrm{b}}$, \\ Matthieu Bec ${ }^{c}$, Maxime Boccas ${ }^{b}$, Camila Duran ${ }^{b}$, Angelic Ebbers ${ }^{b}$, Ariel Lopez ${ }^{b}$, Claudio Marchant ${ }^{b}$, \\ Eduardo Marin ${ }^{\mathrm{b}}$, Vanessa Montes ${ }^{\mathrm{b}}$, Cristian Moreno ${ }^{\mathrm{b}}$, Eric Petit Vega ${ }^{\mathrm{b}}$, Carlos Segura ${ }^{\mathrm{b}}$, Gelys \\ Trancho $^{c}$, Chad Trujillo ${ }^{\mathrm{b}}$, Cristian Urrutia ${ }^{\mathrm{b}}$, Patricio Veliz ${ }^{\mathrm{b}}$, Tomislav Vucina ${ }^{\mathrm{b}}$ \\ aAustralian National University Research School of Astronomy and Astrophysics, \\ Mount Stromlo Observatory, Cotter Road, Weston ACT 2611, Australia; \\ ${ }^{\mathrm{b}}$ Gemini Observatory, AURA casilla 603, Colina el Pino, La Serena, Chile; \\ ${ }^{\mathrm{c}}$ Giant Magellan Telescope Organization Corp., PO box 90933, Pasadena, CA, 91109-0933, USA
}

\begin{abstract}
With two to three deformable mirrors, three Natural Guide Stars (NGS) and five sodium Laser Guide Stars (LGS), the Gemini Multi-Conjugate Adaptive Optics System (Gemini MCAO a.k.a. GeMS) will be the first facility-class MCAO capability to be offered for regular science observations starting in 2013A. The engineering and science commissioning phase of the project was kicked off in January 2011 when the Gemini South Laser Guide Star Facility (GS LGSF) propagated its $50 \mathrm{~W}$ laser above the summit of Cerro Pachón, Chile. GeMS commissioning has proceeded throughout 2011 and the first half of 2012 at a pace of one 6- to 10-night run per month with a 5-month pause during the 2011 Chilean winter.

This paper focuses on the LGSF-side of the project and provides an overview of the LGSF system and subsystems, their top-level specifications, design, integration with the telescope, and performance throughout commissioning and beyond. Subsystems of the GS LGSF include: (i) a diode-pumped solid-state 1.06+1.32 micron sum-frequency laser capable of producing over $50 \mathrm{~W}$ of output power at the sodium wavelength $(589 \mathrm{~nm}$ ); (ii) Beam Transfer Optics (BTO) that transport the $50 \mathrm{~W}$ beam up the telescope, split the beam five-ways and configure the five $10 \mathrm{~W}$ beams for projection by the Laser Launch Telescope (LLT) located behind the Gemini South $8 \mathrm{~m}$ telescope secondary mirror; and (iii) a variety of safety systems to ensure safe laser operations for observatory personnel and equipment, neighbor observatories, as well as passing aircrafts and satellites.
\end{abstract}

Keywords: Multi-Conjugate Adaptive Optics, Laser Guide Star, MCAO, LGS, sodium photon return, LGS spot size

\section{INTRODUCTION}

The Gemini Multi-Conjugate Adaptive Optics (MCAO) System, a.k.a. GeMS, is the first world-wide, facility-class, multi-Laser Guide Star (LGS) MCAO system to be commissioned on a telescope. Feasibility studies for an MCAO capability to be implemented at the Gemini South (GS) 8-meter telescope atop Cerro Pachón in Chile were validated as far back as 1999, and the project passed its Preliminary Design Review (PDR) in 2001. It took no less than a decade for the complex GeMS AO bench (a.k.a. Canopus) and Laser Guide Star Facility (LGSF) subsystems to be built, tested and installed on the Gemini South telescope ${ }^{1,2,3,4,5}$. Both subsystems saw their first laser light ${ }^{6}$ on the Chilean skies on January 22, 2011, when the GS LGSF projected five sodium Laser Guide Stars for the first time in the history of LGS $\mathrm{AO}$ in the presence of a large team of happy scientists, engineers and technicians (see Fig. 1). GeMS commissioning has continued on a monthly basis with 6 to 10-night runs from January 2011 to May 2011, and again from November 2011 to May 2012.

*celine.dorgeville@anu.edu.au; phone +61 26125 6374; fax +61 26125 0233; http://www.mso.anu.edu.com

Adaptive Optics Systems III, edited by Brent L. Ellerbroek, Enrico Marchetti, Jean-Pierre Véran, Proc. of SPIE Vol. 8447, 84471 Q ( ) 2012 SPIE $\cdot$ CCC code: $0277-786 / 12 / \$ 18 \cdot$ doi: $10.1117 / 12.925813$ 
GeMS system performance and demonstrated scientific potential to date are presented in companion papers ${ }^{7}$ and ${ }^{8}$ respectively; this paper concentrates on operational and on-sky results of the GeMS LGS facility. Sections 2,3 and 4 provide an overview of the design, implementation and performance of the main LGSF subsystems, namely the GS laser, Beam Transfer Optics (BTO)/Laser Launch Telescope (LLT), and safety systems respectively. Section 5 describes LGSF system-level, on-sky performance achieved to date, while section 6 presents a brief description of night-time laser operations at the Gemini South Observatory.
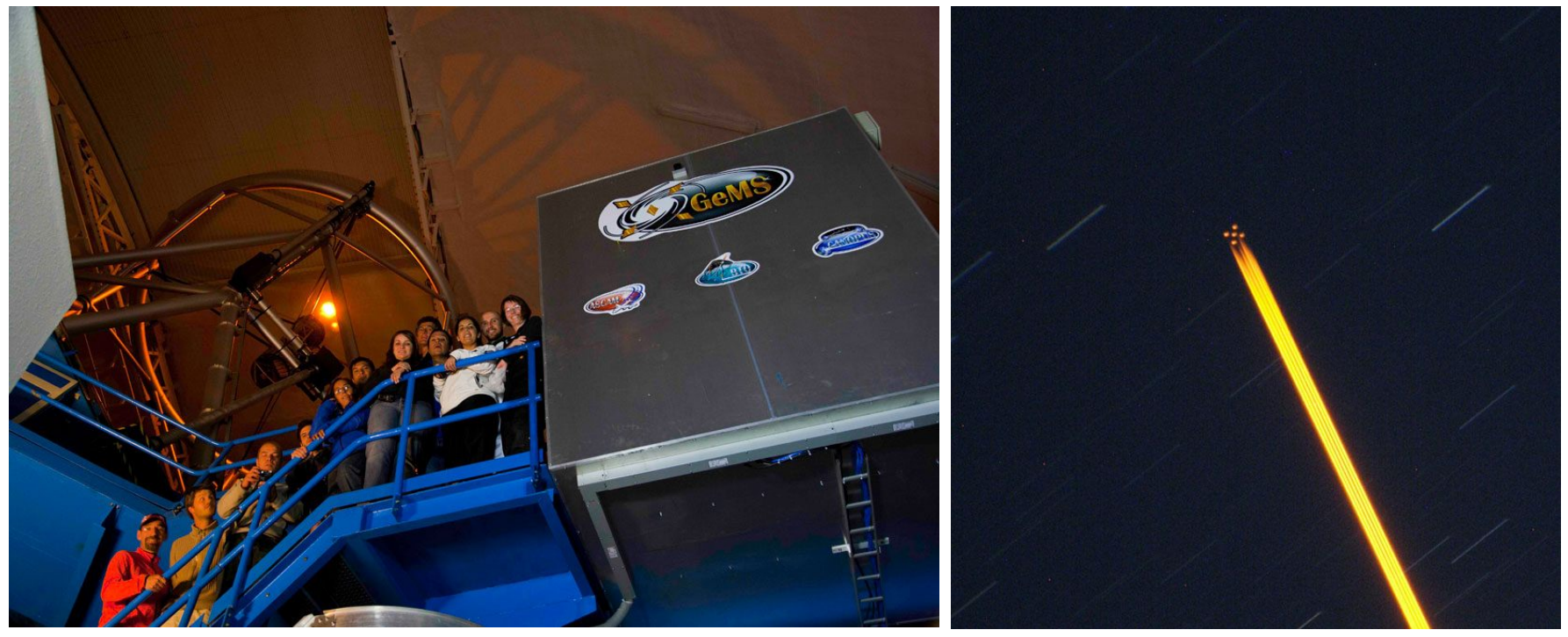

Figure 1: Left: The GS laser was propagated to the dome of the Gemini South telescope for the first time on January 21, 2012. The GeMS commissioning team is seen standing on the stairway leading to the telescope -X elevation platform, next to the GeMS laser room (credit: M. Paredes, Gemini Observatory). Right: The Gemini South LGS constellation was projected to the sky for the first time on January 22, 2012 (credit: M. Boccas, Gemini Observatory).

\section{LASER SYSTEM}

\subsection{History}

The Gemini South Observatory received its 50W GuideStar Lockheed Martin Coherent Technologies (LMCT) sodium laser system ${ }^{9,10,11}$ in March 2010, a key component for GeMS and the most critical component of its LGS facility. After a successful post-delivery acceptance in laboratory, the laser system was installed on the elevation platform of the GS telescope. Following an intensive period of optimization, the GS LGSF had first light on the sky in January 2011. Over the few months following the event, and while the LGSF was being commissioned, the GS laser team gathered laser system performance and operational data, and started to work on ways to improve both overall performance and reliability. This work is critical for the transition of the LGSF system from commissioning to normal operations expected in November 2012.

\subsection{System Overview}

The GS laser contains two infra-red (IR) laser lines (each created by one oscillator and multiple amplifiers), a non-linear stage to combine them, and a set of diagnostics to control the output beam, as shown in Fig. 2. The two Master Oscillators (MOs) operate at $1064 \mathrm{~nm}$ and $1319 \mathrm{~nm}$ respectively. The two MOs, which only differ by the cavity optical coatings, consist of a folded cavity with two birefringence-compensated, diode-end-pumped Nd:YAG rods for the gain heads. The MOs are actively mode-locked using an acoustic-optic modulator, resulting in a $77 \mathrm{MHz}$ pulse train with nominal pulse widths on the order of $\sim 400 \mathrm{ps}$. The relative timing phase between the $1064 \mathrm{~nm}$ and $1319 \mathrm{~nm}$ pulse trains is tunable via the computer control system allowing for the optimization of the pulse temporal overlap in a lithium triborate (LBO) non-linear crystal, to achieve maximum 589nm Sum Frequency Generation (SFG) efficiency. The $1064 \mathrm{~nm}$ oscillator is remotely angle-tuned over $14 \mathrm{GHz}$ with a PZT-controlled etalon to allow the laser frequency to be set at the sodium D2a resonance at any time, using feedback from the wavelength locker module. 
Laser light from the MOs is amplified using a series of LMCT-proprietary planar waveguides each pumped with $300 \mathrm{~W}$, three-bar-diode stacks at $798 \mathrm{~nm}$. At the entrance of the SFG stage, we typically measure $80 \mathrm{~W}$ of light at $1319 \mathrm{~nm}$, and $120 \mathrm{~W}$ at $1064 \mathrm{~nm}$. The $589 \mathrm{~nm}$ beam is then produced with the single-pass focusing of the $1064 \mathrm{~nm}$ and $1319 \mathrm{~nm}$ beams through the LBO crystal. Numerous diagnostic instruments perform a real-time analysis of the laser performance.

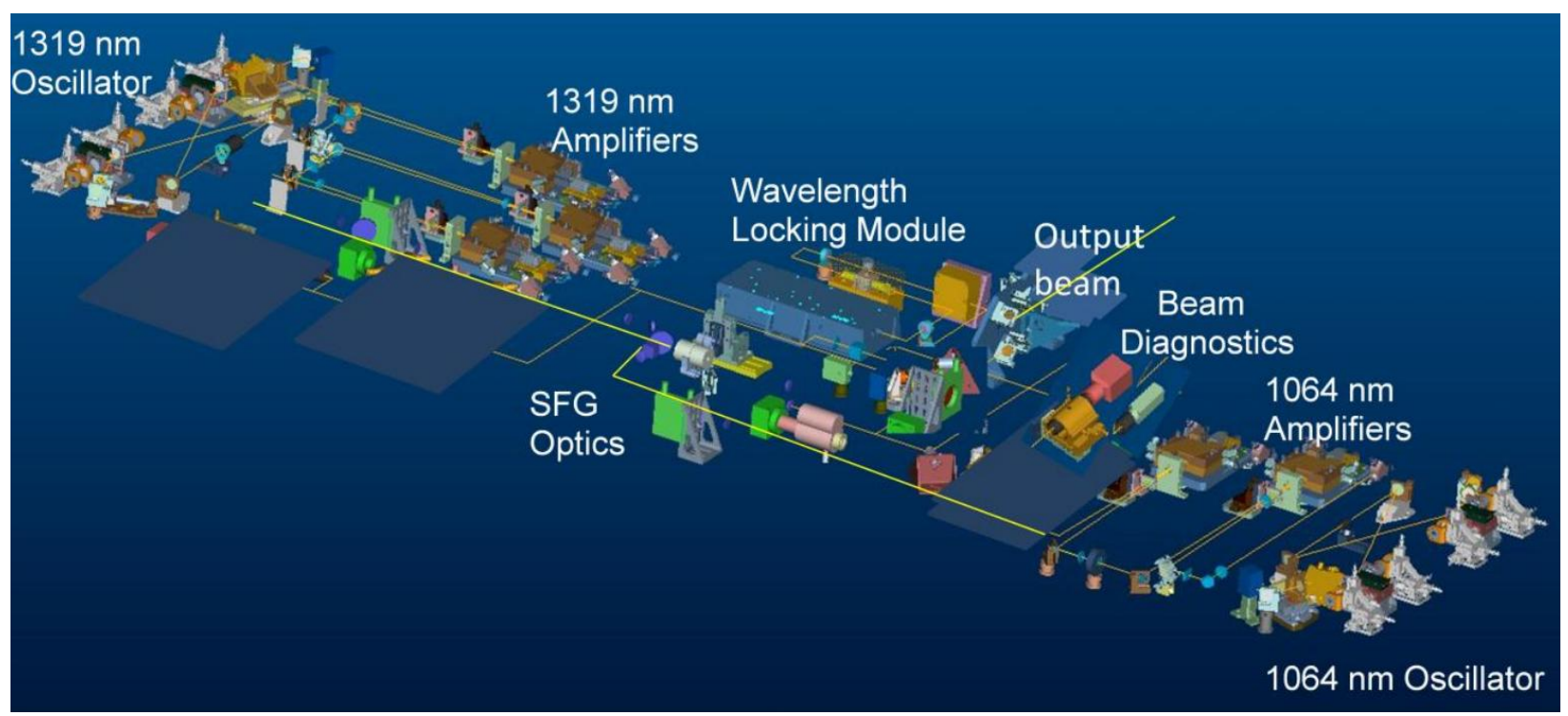

Figure 2: GS Laser System 3D Layout (credit: LMCT)

Table 1: GS Laser output beam requirements and demonstrated performance during acceptance testing

\begin{tabular}{|c|c|c|c|}
\hline Description & Units & Requirement & Measured \\
\hline \hline Average Power & $\mathrm{W}$ & $>50$ & 58 \\
\hline Power Stability - short term Peak to Peak & $\%$ & $<10$ & 16.3 \\
\hline Power Stability - long term RMS & $\%$ & $<5$ & 6.0 \\
\hline $\mathrm{M}^{2}-\mathrm{X}$ & - & $<1.4$ & 1.28 \\
\hline $\mathrm{M}^{2}-\mathrm{Y}$ & - & $<1.4$ & 1.17 \\
\hline Frequency Stability (+/-) & $\mathrm{MHz}$ & $<100$ & 150 \\
\hline Spectral Bandwidth & $\mathrm{GHz}$ & $<2$ & 2.1 \\
\hline Pointing Stability - Transverse - X & $\mathrm{mm}$ & $<0.5$ & 0.006 \\
\hline Pointing Stability - Transverse - Y & $\mathrm{mm}$ & $<0.5$ & 0.006 \\
\hline Point Stability - Angular -X & $\mathrm{urad}$ & $<400$ & 30 \\
\hline Pointing Stability - Angular - Y & $\mathrm{urad}$ & $<400$ & 14 \\
\hline Polarization Contrast Ratio & - & $>20: 1$ & $50: 1$ \\
\hline
\end{tabular}

\subsection{Performance}

Table 1 presents the GS laser requirements and actual performance at the time of factory acceptance testing in January 2010 at the LMCT headquarters near Boulder, Colorado. The GS laser has performed reasonably well after installation on the GS telescope and during on-sky commissioning, but one of its main flaws was and still remains lower than specified output power. Fig. 3 shows that the average power for the November 2011 to May 2012 commissioning period was only about $35 \mathrm{~W}$, making it difficult for the Multi-Conjugate Adaptive Optics instrument (especially in low sodium season) to reach maximum performance. The laser team will dedicate some time during the 2012 GeMS shutdown planned from June to September 2012 in order to improve this parameter. 
However, once the laser is aligned and no change is made that may disturb its environment, the system delivers an output beam with very steady performance for a long period of time. The only issue we have seen is that the average power usually tends to decrease over the length of a run, typically losing around $5 \mathrm{~W}$ over $6-8$ days. We have not been able to correct this trend yet.

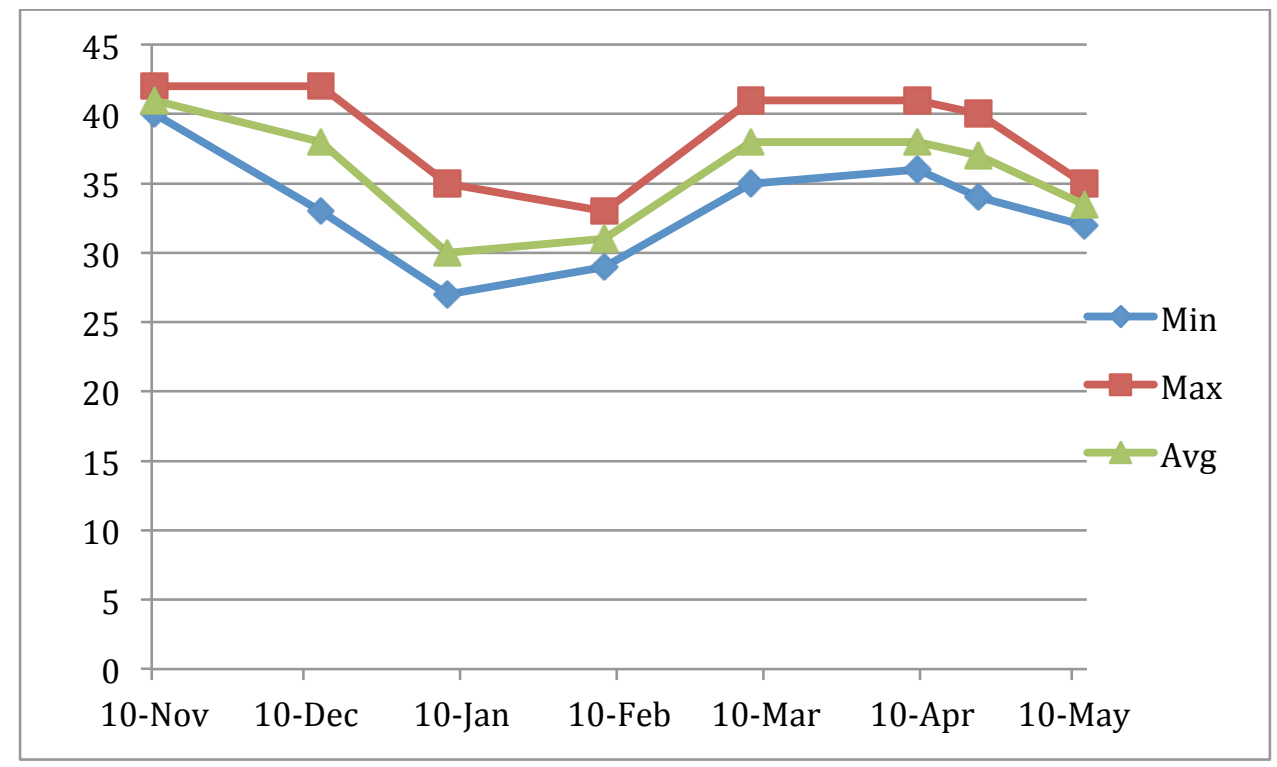

Figure 3: GS laser Average, Min and Max power over a period of 8 months in 2011/2012

Typically, during a laser run, the laser operator only needs to, remotely: (i) perform small adjustments to the non-linear crystal temperature to ensure consistent SFG conversion efficiency; (ii) increase or decrease waveguide amplifier currents to adjust both IR thermal lensing and beam steering for optimum IR beam quality and power; and (iii) adjust the radio-frequency (RF) timing between IR pulses to maximize $589 \mathrm{~nm}$ output power.

The $589 \mathrm{~nm}$ output beam quality is one of the parameters we have learned to improve since the laser was first installed on the telescope. We are now able to deliver a beam with consistent beam quality which, even if not perfect, complies with the original requirements. The laser beam near field output shape varies slightly from run to run, but is considered as a stable parameter in our system (see Fig. 4). Note that the $589 \mathrm{~nm}$ output laser beam quality is highly dependent on the IR laser beam quality input to the SFG stage.

The beam quality $\mathrm{M}$-squared factors $\left(\mathrm{M}^{2}\right)$ in $\mathrm{X}$ and $\mathrm{Y}$ have been measured below 1.5 and 1.3 respectively during the length of the 2011/2012 commissioning campaign. The beam quality is slightly worse in the $\mathrm{X}$-axis because it corresponds to the unguided dimension of the Waveguide Amplifier Module (WAM) amplification stages. We have noticed a slow increase of the $\mathrm{M}^{2}$ values over the length of a run $(\sim 0.1)$, with both axes following the same trend (along with a decrease in power), leading us to think that the system gets misaligned with time. Some ideas to compensate for this behavior have been proposed, but nothing implemented yet.

One surprisingly good aspect of this laser is its wavelength stability. Comparing the Gemini North and Gemini South laser wavelength stability, one can see that the PZT-tuned etalon is a much more efficient and robust technique than the temperature-tuned etalon technique. Corrections are significantly faster, and allow to keep the laser wavelength within a $\pm 100 \mathrm{MHz}$ range centered on the D2a sodium line. Moreover, the wavelength locking process is much easier and faster than it is with the Gemini North laser. 

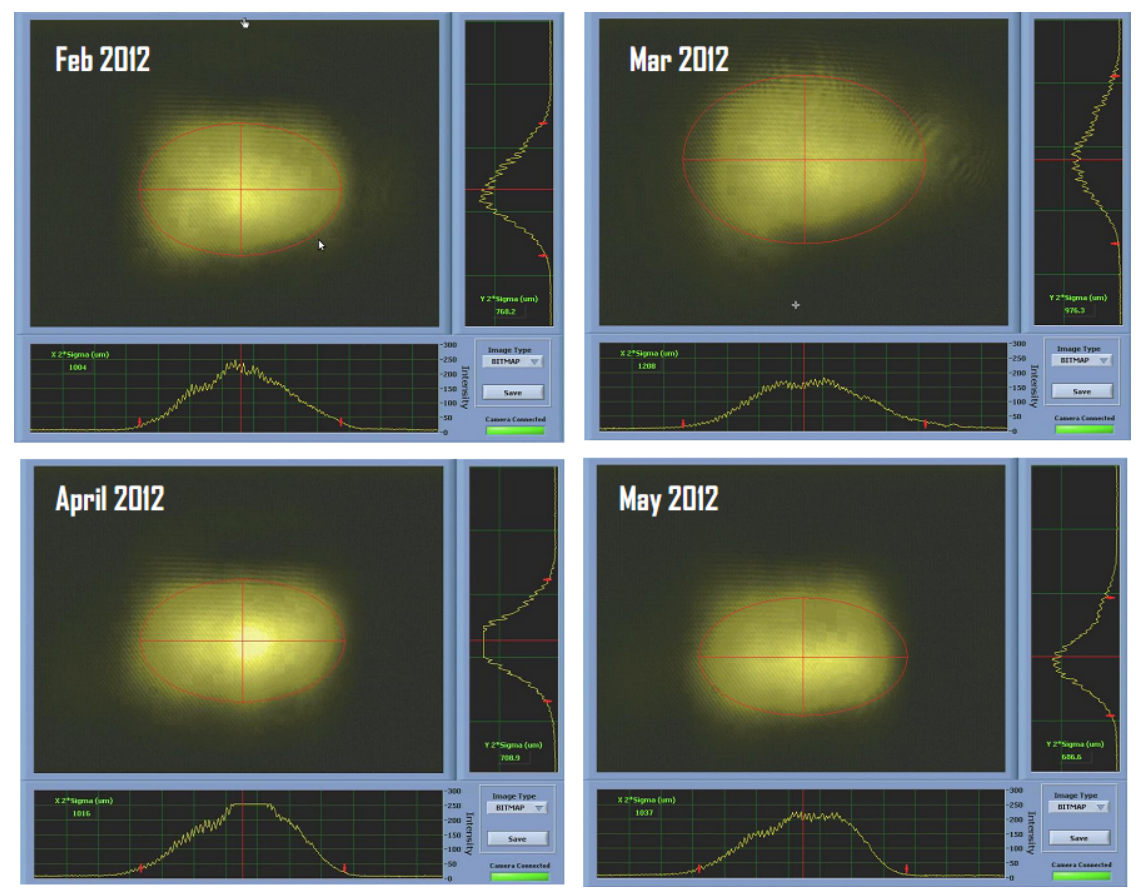

Figure 4: Collimated 589nm beam as seen by the near-field diagnostic camera installed on the laser bench

\subsection{Maintenance}

Monthly maintenance consists mainly in re-doing the SFG co-alignment for both power and beam quality optimization. Indeed, as this optical system is highly subject to temperature hysteresis, as well as telescope vibrations, this critical alignment tends to drift after a while, causing the $589 \mathrm{~nm}$ output beam performance to degrade. Slight adjustments are also performed on the IR lines (before mixing) in order to improve their performance for maximum SFG efficiency.

It is important to note that the laser alignment is highly dependent on controlling the bench temperature to its nominal value (i.e. for the laser running at full power, lids closed, with the laser room temperature at 10C). When some alignment work has to be done on the bench (lids opened), the laser room temperature has to be increased in order for the bench temperatures to match their nominal values. The possibility to change room temperature has made our work much easier and reliable.

Cleaning the oscillator and WAM optics prior to each run also has a significant impact on recovering output power. Critical optics to be cleaned on a regular basis include the laser rods, the LBO crystal surfaces, and the pump diode ferrule ends.

Yearly maintenance consists in a complete cleanup of the system. All the cooling lines are flushed, and their coolants replaced. A full inspection and cleaning of the optical bench is performed as well.

As part of an effort to continuously improve the system performance, the GS laser team now follows a workflow similar to the Gemini North laser workflow that has been delivering good results (see Fig. 5).

Finally, in preparation for the GS laser to move into normal operations, over 50 procedures and processes have been developed and documented. The GS laser master training program requires certification for anyone working on the laser. Both the Senior Laser Technician and Laser Support Engineer provide the training; the Laser Support Engineer issues certifications. 


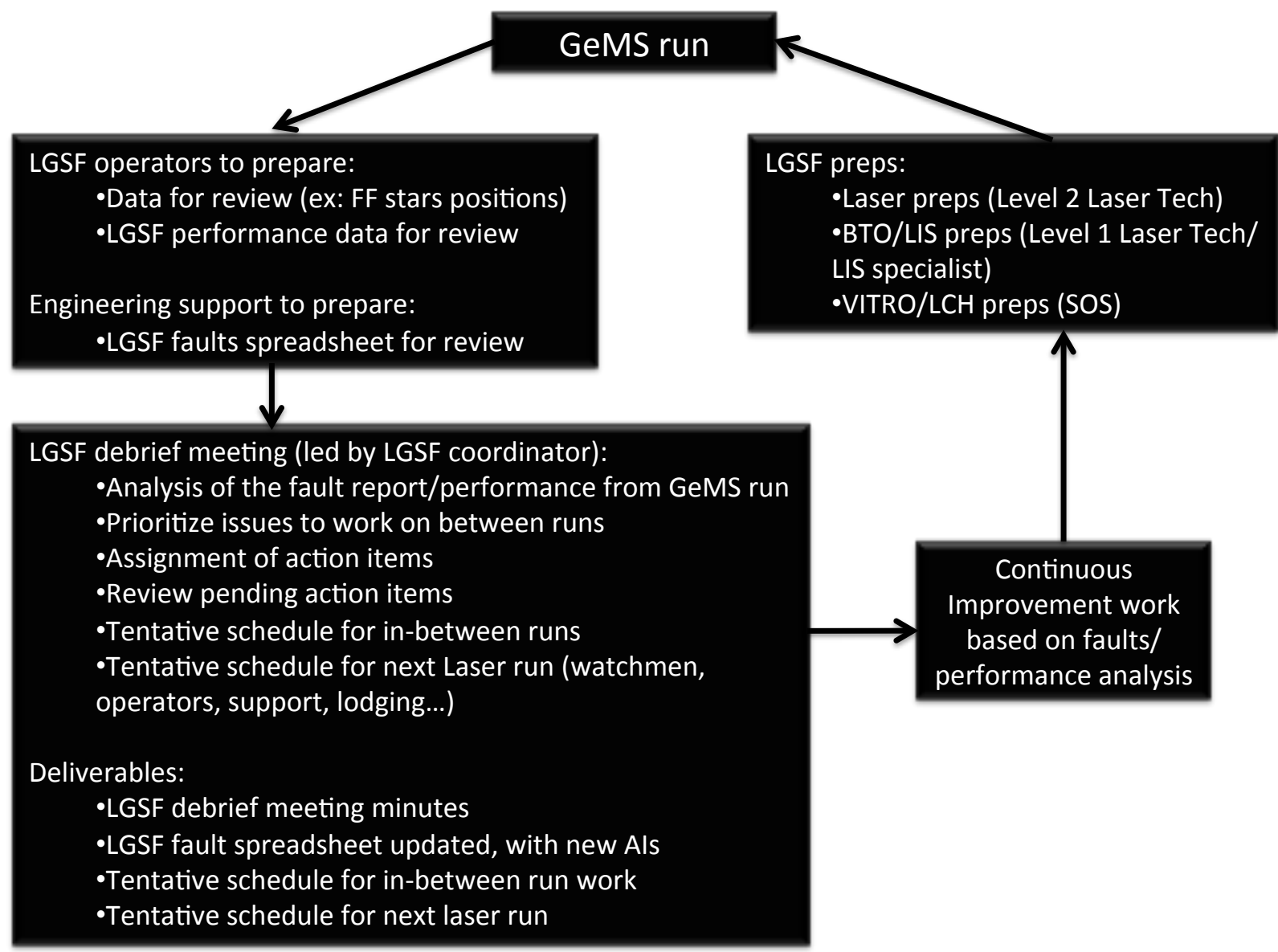

Figure 5: Gemini South laser monthly workflow

\subsection{Upgrades}

Since the laser was delivered at Gemini South, the GeMS laser team has implemented the following system upgrades (see Fig. 6):

- Automated rotation stages for the waveplates located post-amplification in the IR lines allows for remote control of the power sent to the SFG stage. These are used mainly when the laser is left unattended, i.e. when there is a risk of misalignment that could possibly damage a component further down the light path;

- Remote viewing of the three main power meters $(1064 \mathrm{~nm}$ and $1319 \mathrm{~nm}$ post-amplification, and $589 \mathrm{~nm})$ gives the possibility to clearly follow how the system is behaving. For instance, a loss of power on one of the IR lines would immediately be noticed with this setup.

In order to improve both performance and ease of use of the GS laser system additional updates (also shown in Fig. 6) are planned:

- An automated output telescope will allow to remotely launch with precision the laser beam in the BTO without disturbing the very sensitive environment of the laser bench;

- An automated telescope lens positioning, also located near the laser output, will allow to set the collimation lens position with high accuracy in order to optimize laser beam footprints on the LLT and on-sky LGS spot sizes;

- An automated set of mirrors to optimize - probably on a daily basis - SFG co-alignment, will allow to maintain optimum output beam performance during the length of a laser run. 


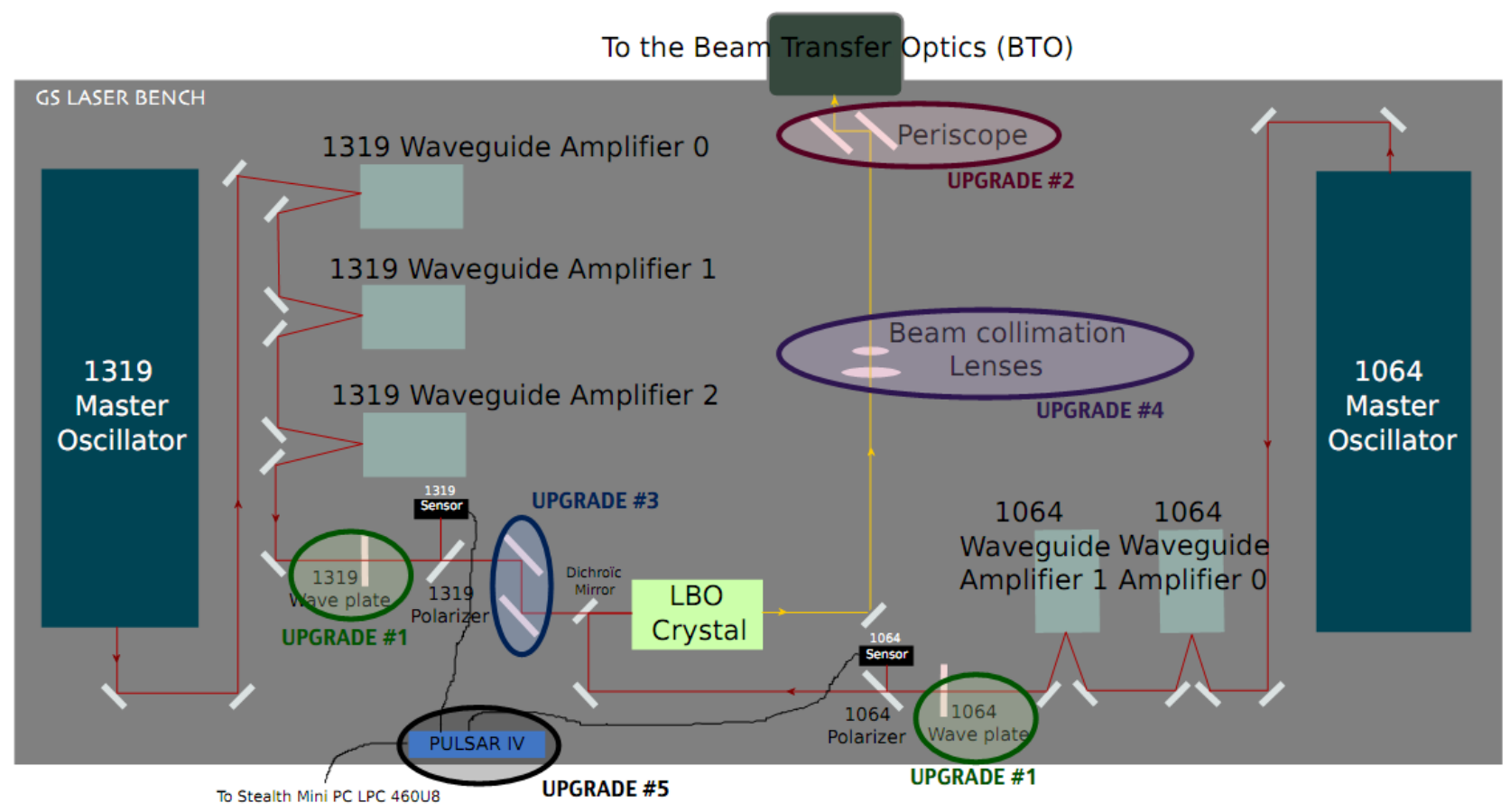

Figure 6: GS laser layout showing the 2011/2012 completed and planned upgrades

The laser team's ultimate goal is to remotely operate the laser from the La Serena base facility during both queue and classical observations. From the time of this writing (June 2012) until October this year, GeMS is in winter shutdown, giving the opportunity for upgrades and performance improvements to be made. In November 2012, the Gemini South laser will move into normal operations. Ongoing improvements will determine when the laser is ready for remote operations.

\section{BEAM TRANSFER OPTICS AND LASER LAUNCH TELESCOPE}

\subsection{Overview}

The Gemini South Beam Transfer Optics (GS BTO) requirements and "baseline design" have been presented in significant details in previous publications ${ }^{2,4}$. The "simplified design" options proposed in $2008^{4}$ have by now been adopted and implemented. As a result, today's GS BTO transports a single $50 \mathrm{~W}$ beam from the GS laser system output up to the top-end ring of the telescope, where the beam is split into five $\sim 10 \mathrm{~W}$ beams prior to projection on the sky by the Laser Launch Telescope (LLT) ${ }^{12}$ located behind the GS telescope secondary mirror. A top-level schematic of the laser beam transport and launch systems is included in the top-level GeMS BTO epics DM screen, illustrating the current laser light path and associated BTO Optical Path (BTOOP), BTO Optical Bench (BTOOB), and LLT components, as shown in Fig. 7. BTO Look Up Table on/off indicators are included on the right-hand side of the screen, and alignment offsets are included next to corresponding actuated components. This screen shot was taken upon powering on the BTO electronics crate, hence the reason why some items are shown in fault/off state (red). When propagating, laser light is drawn in bright orange along the laser path.

\subsection{Throughput}

The GS BTO throughput was measured in early 2011 by propagating the high power $589 \mathrm{~nm}$ laser beam through the BTO and measuring transmitted power at the BTO "beam dump" power meter head located on the BTO Optical Bench (BTOOB) via a "beam dump mirror" (BDM) inserted in the laser path between the BTOOB K-Mirror (KM) and Pointing Mirror (PM). Measurements were calibrated against the GS laser system on-line power monitor, yielding instantaneous throughput readings on the order of $\sim 50 \%$, a very disappointing value with regard to the $75 \%$ that had been expected based on earlier Gemini North BTO results ${ }^{4}$. Because all BTO optics are being inspected and carefully cleaned a couple of days prior to each laser run, in addition to the BTO path being fully enclosed in air-flushed tubing, dust can be ruled 
out as a significant contributor to the low BTO throughput value. Instead this disappointing result is attributed in large part to suboptimal BTO coating specifications that failed to take into account proper polarization control considerations. The worst offender, a low performance aluminum coated mirror mounted at the Truss Pointing Mirror (TPM), was subsequently replaced with a dielectric coated mirror, boosting the BTO throughput up to $\sim 55 \%$, but still falling significantly short of its $75 \%$ specification. Replacement of most BTO optics with better quality coatings and proper polarization control throughout the BTO (not fully commissioned yet) would certainly allow to increase throughput, however personnel resources and BTO access limitations have prevented any significant progress in that area. Future BTO optics upgrades are foreseen in the long term to address this important issue.

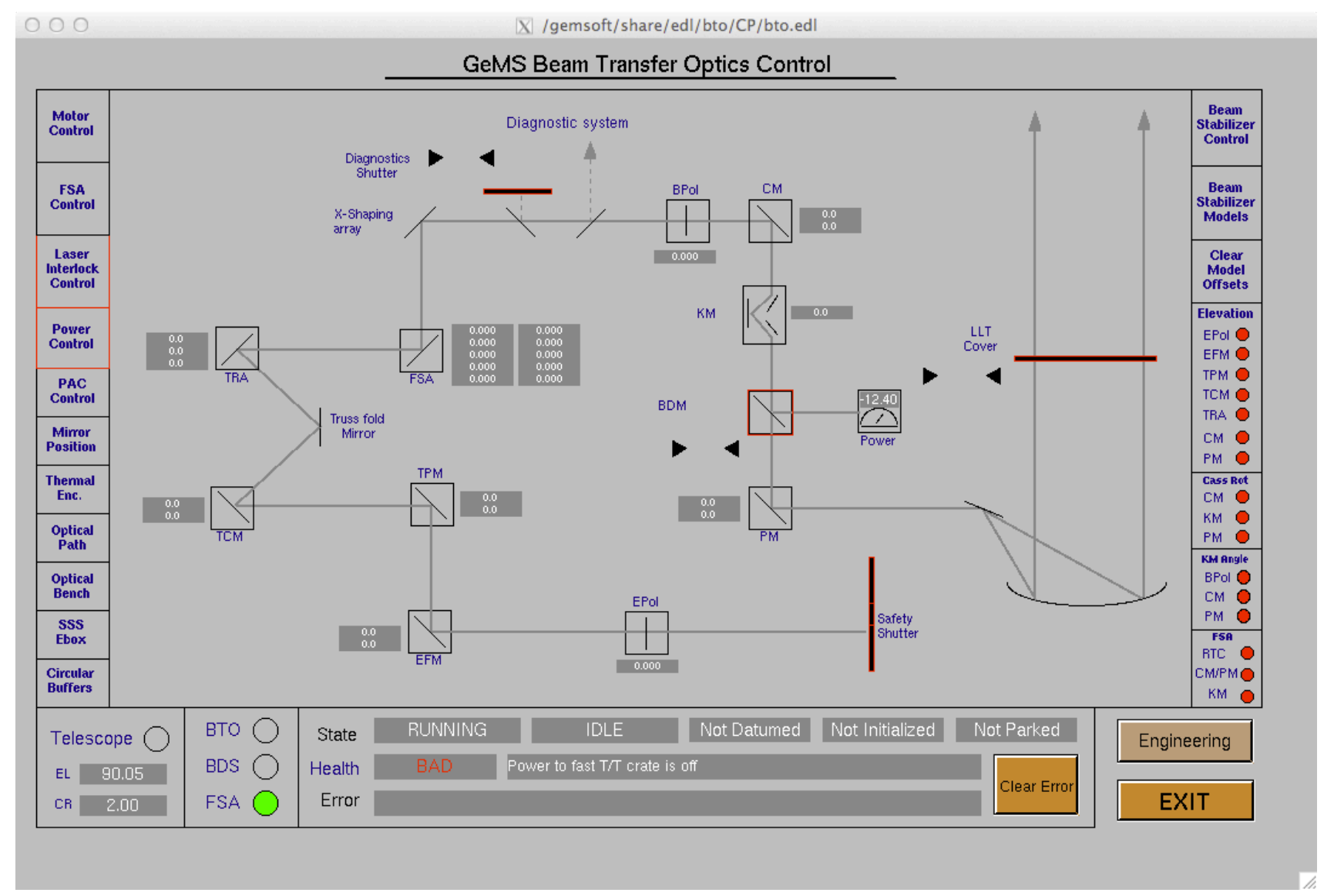

Figure 7: Top-level BTO epics control screen showing the laser light path (grey line) from the safety shutter at the output of the laser system, along the BTO Optical Path (EPol-EFM-TPM-TCM-TFM-TRA), on the BTO Optical Bench (FSAXSA-Bpol-CM-KM-PM), and into the Laser Launch Telescope (LLT).

The LLT was installed on the GS telescope in late $2007^{4}$. Although the LLT cover and primary mirror access ports were kept closed most of the time until laser first light in January 2011, the upward-facing Off-Axis Parabola (OAP) of the LLT collected enough dust to make it easy to visualize laser beam footprints on its surface, at the expense of degraded throughput performance. An in-situ wash of the LLT OAP was performed in December 2011 to recover OAP reflectivity lost to dust. OAP reflectivity coefficients increased from $89.1 \%$ at $530 \mathrm{~nm}$ and $86.0 \%$ at $650 \mathrm{~nm}$, to $98.6 \%$ and $94.8 \%$ respectively, yielding an approximate $10 \%$ reflectivity increase at $589 \mathrm{~nm}$ and bringing the launch system throughput close to its specified $95 \%$.

Based on the BTO and LLT throughput results presented above, we estimate that the LGSF overall throughput is on the order of 50\%. A cleaning maintenance plan needs to be implemented in the long-term to ensure that the LLT throughput does not degrade this already low value significantly over time. 


\subsection{Alignment}

Although the BTO is made of relatively simple optics such as planar mirrors, lenses, beam splitters, and polarization optics, it is a fairly complex system to align due to its large number of optical components that are distributed at odd locations on the Gemini $8 \mathrm{~m}$ telescope. Difficult component access via cherry picker and/or scissor lifts and demanding on-sky pointing accuracy specifications typically require extensive BTO alignment during day-time in-between laser runs and for a few hours at the beginning of each run as well. This section aims to describe the principal steps of the BTO alignment procedure. For obvious safety reasons, all alignments are initially performed at low laser power, either eye-safe below a few milli-Watts or at somewhat higher powers on the order of a few tens of milli-Watts, depending on the application. Laser personnel performing in-situ BTO alignment must wear laser safety goggles at all times.

First, the GS laser system output beam is collimated close to its design $5 \mathrm{~mm}$ diameter at $1 / \mathrm{e}^{2}$ intensity point value, then co-aligned with the elevation axis of the GS telescope using a couple of targets near the laser system output plane and on the Elevation Fold Mirror (EFM). Look-Up Tables (LUTs) vs. elevation are applied to remotely and automatically bring the BTO Optical Path (BTOOP) mirrors to their nominal positions for the current telescope elevation. When pressing the "stabilize" button of the BTOOP stabilization software, feedback from the Truss Point Mirror (TPM), Truss Centering Mirror (TCM), Top-end Ring Array (TRA) and Fast Steering Array (FSA) pre-alignment cameras (PACs) is processed in order to provide closed-loop control of the EFM, TPM, TCM and TRA actuators so as to further optimize beam centering on each mirror. Optimized positions are saved for all actuators and the corresponding LUT models are updated accordingly. Note that "stabilize" adjustments can only be performed at low power due to the limited dynamic range of the pre-alignment cameras, thus currently precluding closed-loop BTOOP alignment during on-sky laser propagation at high power.

The next BTO alignment step consists in aligning the LGS constellation to its nominal position. Currently this alignment can only be performed in-situ via manual tip/tilt adjustments of each of the five FSA and five XSA (X-Shaping Array) mirrors. Access to the BTOOB components either require that (i) the telescope be positioned at $\sim 20$ degree elevation and pointed near the dome balcony, from where laser personnel can reach the FSA and XSA inside the BTOOB; or that (ii) the telescope remain at zenith and personnel make use of the cherry picker to access the very top of the telescope. The first option was favored initially for reasons of telescope and personnel safety (maneuvers near the secondary mirror of the telescope with the cherry picker have proven to be extremely risky, both for hardware and personnel). However nonelastic mechanical flexures in the BTO made it so difficult for the LGS constellation alignment to reach the required one arcsecond LGS constellation on-sky relative pointing accuracy that the second option has ultimately been favored instead. Due to the difficult BTOOB access constraints, recent LGS constellation alignments typically require several hours of day-time, closed-dome alignment prior to a laser run. They also require a couple of hours of night-time, open dome alignment at the beginning of the run, when direct feedback from the GeMS LGS Wave Front Sensors (WFS) is required to align each LGS on its corresponding WFS with sub-arcsecond accuracy.

Another critical, manual BTOOB alignment consists in ensuring that the on-axis laser beam reflecting off the BTOOB Centering Mirror (CM) is precisely co-aligned with the BTOOB K-Mirror rotation axis at all times so that KM does not introduce any vignetting or unwanted beam motion on the LLT primary-mirror (the OAP is the optical stop of the BTO/LLT optical train). This condition was not initially met during the 2011 GeMS commissioning phase and effectively resulted in poor pointing performance of the PM/CM LUTs vs. elevation, KM angle, and Cassegrain Rotator (CR) angle when propagating the laser on-sky. A recent thorough realignment of the BTOOB with the telescope pointing at zenith seems to have corrected this problem, bringing the LGSF blind pointing accuracy (with all LUTs applied) from tens of arcseconds down to a couple of arcseconds. The LGSF blind pointing performance is now close enough to its original one arcsecond specification so as to enable nearly instantaneous LGS acquisition on the GeMS LGS WFS.

\subsection{Control System Reliability}

The BTO/FSA controller utilizes two Emerson MVME6100 processors on a common VME backplane, one dedicated to low bandwidth BTO mirror train control and EPICS interface, and one dedicated to high-bandwidth FSA control. These two processors communicate via a VxWorks shared memory communications protocol. During the early commissioning phases this control system suffered from significant stability issues: in seemingly normal operation the BTO/FSA controller would spontaneously "crash" after a few hours of operation. Recovery from a system crash required a full system re-boot and reload of the operating program. Each system crash resulted in at least 20 minutes lost observing 
time. Since the system crashes were apparently random and were not preceded by a consistent set of symptoms, considerable effort was required to successfully resolve this issue.

During a period of rigorous code review and systematic troubleshooting a number of contributing factors were discovered and rectified, for example: messages passed over the shared memory mailbox system were not checked for size, resulting in buffer overflows which overwrote critical operating system areas; VxWorks tasks that performed floating point operations were not always created with the correct floating point options; and interrupt service routines were not optimized, especially those relating to the reflective memory and motion control cards. A related hardware issue in which the VME interrupt acknowledge and bus grant chains were not connected correctly on custom interface boards was also identified and resolved. The detailed system software analysis led to many critical tasks and processes being re-coded to increase operator efficiency and improve overall system performance. As a result of this work the BTO/FSA controller operated continuously during the last two commissioning runs with no sign of instability or performance degradation.

\subsection{Lessons Learned}

So many lessons have been learned during the 2011A, B and 2012A GeMS commissioning phase about the BTO and LLT subsystems that it is unfortunately not possible to present and detail them all in the present paper. This section will focus instead on lessons learned that might provide useful design and operation experience to existing and future LGS facilities.

The very first lesson, which seems quite obvious, is that the BTO optical train should be kept as short as possible. The price to pay for a short BTO is either to project laser beams off-axis from multiple launch telescopes (the higher LLT cost more or less balance the lower BTO cost), or to launch laser beams on-axis and mount the laser system (or at least its last stage, often called the laser head) behind the telescope secondary mirror. Gemini Observatory made the choice of a single, on-axis launch at the very start of the MCAO design phase so as to reap as many benefits from the Gemini North and South LGSF commonalities as possible, based on identical LLTs and similar BTOs. The GS laser system had to be mounted off-telescope for technological and contractual reasons, resulting in an unfortunately long GS BTO. Long BTOs are complex systems, involving a large number of surfaces (resulting in undesirably low throughput given how expensive laser photons are), a large number of controlled elements (more electronics and software), and generally resulting in significantly higher design and fabrication costs as well as higher failure rates than short BTOs. Existing and future AO projects involving large LGS facilities should certainly ponder the cost and operation benefit trade-offs of long vs. short BTOs before choosing one over the other.

Another important lesson to learn from our GeMS experience is that AO projects should pay close attention to the choice of BTO optics and their optical coatings. As mentioned earlier, $589 \mathrm{~nm}$ laser photons are extremely expensive, on the order of 50,000 to 250,000 USD per Watt of laser system output power. Thus it is certainly cost effective to invest in quality BTO optics in order to maximize throughput and enable good polarization control so as to save precious laser photons (circular polarized beams have the capability to increase sodium photon return by factors up to three ${ }^{13}$ depending on the laser spectro-temporal format and observatory site).

We have also learned a good many lessons regarding the location and use of piezo-driven fast tip/tilt platforms to compensate for atmospheric turbulence jitter of the LGS and stabilize them on their respective LGS WFS. First, the BTO optical design should ensure that fast tip/tilt mirrors (FSM) or arrays (FSA) are located in a pupil plane of the LLT (assuming that the LLT primary mirror is the optical stop of the system). When they are not, as is the case for GeMS, laser beam footprints will continuously move on the LLT primary mirror and risk being vignetted. Not only will projected laser power and spot size per LGS vary over time, but the Rayleigh beam footprints on each LGS WFS (called fratricide) will also change rapidly, destroying any hope to ever subtract the Rayleigh background from the LGS WFS frames as well as creating all sorts of spurious effects for the AO reconstructor. Second, fast piezo platforms should be selected (if possible) to provide more dynamic range than just what is required to compensate for atmospheric turbulence, as their dynamic range is likely to be called upon to compensate other optical misalignments in the BTO and AO optical systems (for instance LGS constellation alignment to the GeMS LGS WFS). Third, close attention should be paid to their mechanical mounting making sure that manufacturer recommendations are heeded (e.g. no clamping of the piezo body). And fourth, great care must be taken in their computer control (see comments on the FSA "control system reliability" in section 3.4 above). 
If a K-Mirror (KM) is required to rotate the LGS constellation on-sky, the optical design should also ensure that the laser beam pointing into the KM is fixed no matter what the telescope elevation is. This is not the case in the GS BTO, where (for space envelope constraint reasons) the KM is currently located between the Centering Mirror (CM) and Pointing Mirror (PM) on the BTO Optical Bench (BTOOB). This unfortunate arrangement initially led to the GeMS LGSF blind pointing issues already discussed in section 3.3. As a result, automatic pointing adjustments made by the PM/CM LookUp Tables (LUT) vs. KM angle would not be repeatable between one telescope elevation pointing and the next, either due to necessary, post-slew BTO Optical Path (BTOOP) realignments that effectively modified pointing inside the BTOOB, and/or due to the PM/CM LUT vs. elevation that was meant to compensate telescope flexures but also modified CM pointing into the KM.

Examples of additional lessons learned include, in no particular order: turning off the air inside the enclosed BTO path during night operations so as not to introduce unwanted beam jitter (but turning air back on during day time so as to maintain positive pressure and keep dust out); aligning the BTO preferably at zenith instead of aligning with the telescope positioned at the more convenient 20 degree elevation because it is not close enough to typical, night-time telescope elevation pointing to ensure adequate LGS constellation alignment; thoroughly testing LLT image quality throughout the temperature range as well as rate of change expected at the telescope; preventing local turbulence from building up in the LLT structure; etc.

\subsection{Past and Future Upgrades}

At the beginning of each laser run, the first high power laser propagation to the sky is typically followed by a LLT focus run in order to minimize the LGS spot size as seen by the Gemini telescope acquisition camera (with the telescope defocused to the sodium layer altitude) or by the LGS WFS (with the telescope focused at infinity). The LLT focus control automation upgrade was probably the most successful among the few BTO and LLT upgrades that were implemented during the GeMS 2011 winter shutdown. Installing a remotely controlled focus screw on the manual LLT focus adjustment knob has made it possible to optimally focus the LLT (and optimize the LGS spot size) within a matter of minutes instead of hours. Prior to that upgrade, LLT focus adjustment was performed by a (reasonably tall) optical engineer standing on a scissor lift extended to its maximum height so as to reach the back of the LLT mounted behind the secondary mirror with the telescope pointed near 20 degree elevation. In order to reach optimum LLT focus, a fairly large number of iterations had to be performed between zenith and 20 degree. Not only did this exercise take significant time at night, but it was also relatively risky and quite uncomfortable for the two people standing high above the floor in the cold and dark Gemini South dome.

Software upgrades have been implemented throughout the 2011 and 2012A commissioning period not only to fix BTO software bugs at high level (EPICS) as well as low level (motion control) but also to improve Graphic User Interfaces (GUI). Simpler, more concise EPICS DM screens have been created to allow the laser operator to control all the BTOOP/BTOOB LUTs and diagnostics from a minimal number of screens. The PM/CM LUTs vs. KM angle and Cassegrain Rotator (CR) angle were simplified to include only the LUT vs. KM angle while forcing the KM angle to follow the CR angle at all times. Laser alignment in the BTO and LGS pointing on-sky is also now better interfaced with other GeMS control features such as on-sky LGS constellation alignment and FSA closed loop control.

Another upgrade of sorts that was recently adopted was to decommission (or rather fail to commission) the Laser Bench Beam Stabilization system (LBBS) presented in ${ }^{4}$. Real-time stabilization of the 50W laser beam between the Truss Pointing Mirror (TPM) and the Top-end Ring Array (TRA) seems not to be required for the BTO to perform reasonably well. The LBBS was indeed rather advantageously replaced by the development and punctual use of the "stabilization" software to optimize BTOOP alignment prior to propagation of the 50W laser at high power in the BTO. Planned upgrades to this capability during the GeMS 2012 winter shutdown include retrofitting the BTOOP Pre-Alignment Cameras (PACs) with remotely controlled neutral density filter holders so that "stabilization" can be performed at high power as well as low power, possibly in permanent closed loop control.

Finally, other desirable, longer-term upgrades include: modification of the XSA mount to enable remote alignment of the LGS constellation on the GeMS LGS WFS following initial laser propagation at the beginning of each laser run; replacement of low-throughput, polarization-scrambling BTO optics; and refinement of the BTO polarization control scheme. 


\section{SAFETY SYSTEMS}

\subsection{Overview}

The Gemini South laser safety systems were designed as the result of a laser safety hazard analysis performed by the GeMS design team in compliance with American National Safety Standards (ANSI) Z136.1-2007 ${ }^{14}$ and Z136.6-2005 ${ }^{15}$. Laser safety systems associated with safe indoor operation and outdoor propagation of the class IV GS laser at the Gemini South Observatory have been described in details in ${ }^{4}$. They include a Laser Interlock System (LIS), aircraft and satellite avoidance systems, and a beam collision avoidance system called the "Laser Traffic Control System". The commissioning status of each of these systems is presented below.

\subsection{Laser Interlock System}

The Laser Interlock System (LIS) is an Alan Bradley Programmable Logic Controller (PLC)-based system that lies at the heart of the LGSF safety systems. The LIS receives inputs from all GeMS subsystems as well as other observatory systems (e.g. earthquake detector, telescope control system, etc.) and processes them in real-time. Should the LIS detect a laser condition that is unsafe for personnel and/or hardware, an interlock sequence is triggered that results in shuttering of the laser beam(s) either at the laser system output or at the BTO shutter. The most unsafe conditions (for instance fault state of the safety shutter) will trigger an emergency shutdown of the GS laser system itself.

The most critical LIS integration and tests were performed in advance of the January 2011 laser first light and under the vigilance of the Gemini Laser Safety Officer so as to ensure safe laser operations at all times. Debugging of the LIS shuttering sequences required running many systems in simulation mode, either when these systems were not available, or because the results of the interlock sequences were undesirable. Laser emergency shutdowns for instance will invariably result in laser misalignment and associated laser system performance issues; at worst they will cause damage to expensive laser diode equipment. Time to recover from laser emergency shutdowns vary from a few hours to a few weeks depending on the extent of the damage incurred. After the GS laser system incurred several emergency shutdowns, most of them caused by negligence or poor coordination during the LGSF integration and test phase, it was decided that the LIS "emergency shutdown request" output to the GS laser system would be bypassed until GeMS commissioning were complete.

Although LIS testing of all inputs and outputs could be performed during day time, full commissioning of the LIS eventually required night time propagation and testing in real operational conditions. LIS "pause" and "resume" sequences following early detection of an aircraft by spotters (see section 4.3 below) were for instance implemented and commissioned to aid in streamlining night-time laser operations. Since January 2011 the Gemini South Observatory has incurred various minor earthquakes that did not disturb GeMS commissioning or result in significant LGSF misalignment. However when a larger, magnitude 5.6 earthquake hit the summit during on-sky laser propagation on April 30, 2012, the observatory earthquake sensor actually failed to detect it and it was the laser operator who immediately closed the safety shutter and proceeded to shutdown the laser system. Fortunately no damage occurred. Tuning of the earthquake sensor detection threshold is obviously required and will be performed during the 2012 GeMS winter shutdown.

\subsection{Aircraft and Satellite Avoidance}

The aircraft avoidance regulations that Gemini follows originate from the American National Standards Institute ${ }^{15}$, the Federal Aviation Administration (FAA) ${ }^{16}$, and also from an agreement with the DGAC, or Dirección General de Aeronáutica Civil de Chile, Chile's equivalent of the FAA. Predictive satellite avoidance is used to prevent damaging sensitive satellite instruments with pre-approval of targets via the U.S. Space Command Laser Clearing House (LCH). While propagating, the laser and surrounding sky are monitored by both software and personnel at all times for any nearby aircraft and satellites.

\section{Aircraft Avoidance}

The Santiago International Airport, located approximately 220 miles South of Cerro Pachón, is a major hub for international flights to South America. Due to the geography of the country, many arrivals and departures have flight paths that pass near and sometimes directly over the observatory. Thus to avoid any aircraft illumination Gemini has trained a team of laser spotters. On any given laser night, there are always three laser spotters at the observatory. 
Spotter 1 is located outside, well away from the observatory giving a clear vantage point of the sky to monitor the laser and surrounding area for any approaching planes. Only with this spotter's permission is the telescope operator allowed to propagate the laser on-sky. If an approaching aircraft will pass within a radius of 25 degrees of the laser (named the safety zone), the spotter outside will make radio contact with the operator and the laser will be shuttered. In the event that radio communication cannot be made, the spotter has a button, cleverly named the "spotter button", with a hard connection to the laser safety shutter that will both shutter the laser and interlock the system. Until radio communication is reestablished the interlock cannot be reset and the laser cannot be propagated to sky.

In addition to the outside spotter, spotter 2 is seated near the telescope operator in the control room, but at a separate computer station monitoring the VITRO system (Sistema de Visualización de Tránsito Aéreo). VITRO is a web-based radar map of the area showing a real-time feed of aircraft locations, altitudes, velocities and flight directions near La Serena. Spotter 1 and 2 are in constant radio communication when aircrafts are present, and maintain logs of all spotted aircrafts.

To reduce both eye and general fatigue, spotter 3 is on break. The spotter team is on a one-hour rotation which includes 10 minutes of overlap time outside for dark adapting.

In practice the spotter and telescope operators work very well together, and the spotters are able to give the telescope operators a warning several minutes in advance if an aircraft is approaching, allowing the telescope operator to shutter the laser more conveniently.

\section{Satellite Avoidance}

In addition to aircraft illumination avoidance, Gemini also uses predictive avoidance for satellites. A week before each laser run, Gemini sends a Predictive Avoidance Request Message (PRM) to the Laser Clearing House (LCH) with the RA and Dec of each target we wish to observe. These targets are then processed by the LCH and returned to Gemini ahead of the run in a Predictive Avoidance Message file (PAM) showing the allowed times of propagation for each requested telescope position. When a satellite passes within the laser avoidance cone which includes a 0.25 degree halfangle for laser pointing uncertainties and additional, unknown margin defined by the LCH, a shutter window will be shown in the PAM.

This file is ingested by a software tool that provides a countdown to all shutter windows that match the current telescope position (in RA/DEC), and sends a signal to the LIS to safely shutter the laser 10 seconds before the actual event. In addition to this software tool, the operator and spotter 2 on the VITRO station are also vigilantly watching for shutter windows.

\section{Shutter Statistics and Future}

Over a total of 13 laser commissioning nights in February and March 2012, statistics were compiled on the amount of time we spent shuttered due to aircraft or satellite events (Table 2). The time spent shuttered was defined as the time it took for operations to return to its previous state. For instance, a shutter for a plane passing through the 25 degree safety zone may take only 30 seconds, but for operations to return to the previous state of propagating and offloading corrections to the telescope it may take an additional 2 minutes. Thus all of this was counted as time spent shuttered. In the future as the operators become more familiar with the system it is expected that this additional time lost will be minimal. Note that the "\% of Time Shuttered" is actually a lower limit, as the "Time on Sky" counted the maximum possible laser time on sky, and did not factor in time spent not propagating due to faults or non-laser commissioning tasks during those nights.

In the future it is Gemini's plan to move to a fully automated aircraft and satellite avoidance system. The software for this system is currently in development. 
Table 2: February and March 2012 shutter statistics

\begin{tabular}{|l|l|}
\hline Time on Sky & $102.1(\mathrm{hr})$ \\
\hline Time Shuttered to PLANES & $2.54(\mathrm{hr})$ \\
\hline Time Shuttered to LCH & $1.99(\mathrm{hr})$ \\
\hline Total Time Shuttered & $4.53(\mathrm{hr})$ \\
\hline$\%$ of Time Shuttered & $4.44 \%$ \\
\hline
\end{tabular}

\subsection{Beam Collision Avoidance}

While Gemini uses laser guide stars at $589 \mathrm{~nm}$ for guiding and high-order corrections, this also implies that other telescopes observing in this wavelength regime can image the $589 \mathrm{~nm}$ light. Thus, to prevent impacting science observations from other observatories, the Laser Traffic Control System (LTCS), a system initially designed for use on Mauna $\mathrm{Kea}^{17}$, was adapted for use on Cerro Pachón.

Each telescope publishes its current pointing data in a URL format that is read-in by the LTCS code. The LTCS then calculates if any beam collision will occur between observatories based on a geometric model of the telescopes and their pointing field of views. If a collision between any two telescopes will occur, the LTCS provides a visual countdown to the beam collision via the "Status and Alarm Summary" screen (Fig. 8) and eventually when the collision occurs will shutter the laser via the LIS. While the pointing data of telescopes is not available to the operators, the LTCS has an easily readable GUI that will show telescope operators at once if a beam collision will occur.

If a beam collision is predicted but the observations are not laser sensitive, the LTCS can be overridden (with a phone call confirmation) allowing laser operations to proceed in the area. Below is an example of the LTCS Status and Alarms Summary, the GUI that the telescope operators use to determine if a beam collision will occur.

As shown in Fig. 8, Gemini, the Southern Astrophysical Research telescope (SOAR) and the Andes Lidar Observatory (ALO) are currently the only observatories included on the Cerro Pachón LTCS. A study is being conducted at the Cerro Tololo Inter-American Observatory (CTIO) to determine whether the CTIO science can also be impacted by our laser. In the future it is expected that the Large Synoptic Survey Telescope (LSST), which will also be located on Cerro Pachón, will be added to this system.

\section{Status \& Alarms Summary}

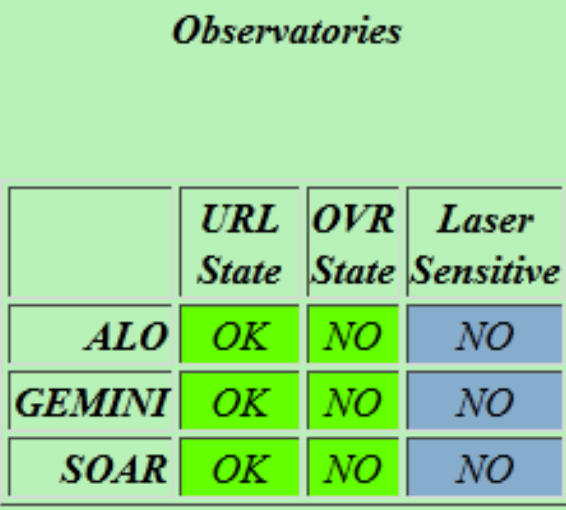

\begin{tabular}{|c|c|c|c|c|}
\hline \multicolumn{5}{|c|}{ Lasers } \\
\hline & Laser State & $\begin{array}{l}\text { Shutter Event } \\
\text { (site,duration) }\end{array}$ & \multicolumn{2}{|c|}{$\begin{array}{c}\text { Predictions } \\
\text { (number, site list) }\end{array}$} \\
\hline GEMINI & $\mathrm{O} n-\mathrm{Sk} y$ & None & \multicolumn{2}{|c|}{ None } \\
\hline$S O A R$ & Off & None & \multicolumn{2}{|r|}{ None } \\
\hline \multicolumn{5}{|c|}{ Collisions } \\
\hline Laser & Scope & Started & nds & Priority \\
\hline \multicolumn{5}{|c|}{ Laser "ON" Preview (Predictions \& Collisions) } \\
\hline Laser & Scope & Starts & ids & Priority \\
\hline
\end{tabular}

Figure 8: Cerro Pachón Laser Traffic Control System "Status and Alarm Summary” screen 


\section{LASER GUIDE STAR FACILITY ON-SKY RESULTS}

\subsection{LGSF Commissioning Overview}

The Gemini South LGS Facility propagated its laser for the first time above Cerro Pachón about a year and half ago on January $22,2011^{6}$. The January 2011 run (4 nights) was entirely dedicated to LGSF commissioning, while subsequent runs offered a mix of MCAO and LGSF commissioning tasks, depending on conditions such as weather, system failures, support staff availability, as well as progress made with the GeMS commissioning plan.

The first and probably most important lesson to be learned from our GeMS experience is that the LGS facility of a complex AO system such as the Gemini MCAO System is to be considered an instrument in its own right, requiring substantial dedicated day and most importantly night engineering and commissioning time. We estimate that LGSFspecific commissioning tasks have thus far used as much of 20 to 30 nights out of the total 81 nights allocated to GeMS commissioning since laser first light in January 2011 (see Table 1 in ${ }^{7}$ ). Most of the on-sky LGSF commissioning time was spent either focusing the Laser Launch Telescope to achieve the smallest possible LGS spots on the sodium layer (see section 5.3 below), aligning the LGS constellation on the GeMS LGS WFS, or taking data for PM/CM Look-Up Tables (LUTs) and testing them. Several LGSF commissioning results typically came as a by-product of other MCAO commissioning activities, for instance sodium photon return data (presented in section 5.2 below), fast LGS jitter compensation (presented in section 5.4 below) and general LGSF reliability data (e.g. BTO control system instability issues discussed in section 3.4, now fixed). Propagating the GS laser on the sky also involved continuous testing of the LGSF safety systems in real-time, such as propagation interrupts by the Laser Interlock System (LIS) in case of aircraft/satellite detection (section 4.2) or beam collision with SOAR (section 4.3), and emergency system shutdowns caused by laser cooling system flow faults, to name just a few.

In the following sections we present the Gemini South LGSF system-level, on-sky performance results based on data gathered since laser first light.

\subsection{Sodium Photon Return}

Tables 3, 4 and 5 below present a summary of the sodium photon return results obtained on Cerro Pachón when propagating the GS laser during the period November 2011 through May 2012. These results, as well as additional results obtained earlier in 2011A are described in much greater details in a companion paper ${ }^{18}$. We focus here on comparing the results presented in Table 1 of ${ }^{18}$ with the sodium photon return specification that had originally been developed for the MCAO Preliminary Design Review (PDR) in $2001^{2}$.

An important outcome of the 2001 MCAO PDR was the specification of the output power level required for the $589 \mathrm{~nm}$ GS laser system. It was believed at the time that, to achieve the 250 to 390 photons per subaperture per frame required on each of the five LGS WFS for MCAO to perform at its science specification level, five 10W-class LGS were required. Cost and technical risk considerations were weighted against acceptably low system margins, leading to the decision to procure a $50 \mathrm{~W}$ laser system with either a $\mathrm{CW}, \mathrm{CW}$ mode-locked or macro-micro-pulse laser format. It was clear from the start that a 50W laser system would offer little to no margin in terms of LGS brightness; the mitigation strategy would be to accept degraded performance or limit MCAO observations during times of higher sodium abundance.

Table 3 provides a summary of the various 2001 assumptions that were made regarding transmission factors to convert laser photons into photo-detection events at the LGS WFS, as well as 2011-2012 estimates based on measurements and/or educated guesses for the same quantities. As can readily be seen in this table, the overall GeMS (LGSF + telescope $+\mathrm{AO}$ ) system throughput falls short of the original specification by a factor of nearly 2 .

In Table 4, photo-detection events are converted in photon flux at the LGS WFS using the design parameter values adopted for MCAO frame rate and subaperture area, and in photon flux at the primary mirror of the telescope based on the 2001 assumptions presented in Table 4. The resulting GS LGSF sodium photon return specification per LGS is in the range of 166 to 257 photons $/ \mathrm{cm}^{2} / \mathrm{s}$ at the primary mirror of the telescope, corresponding to a range of 23 to 36 photons $/ \mathrm{cm}^{2} / \mathrm{s} / \mathrm{W}$ of projected laser power per LGS (i.e. $7.2 \mathrm{~W}$ based on 2001 assumptions listed in Table 3). 
Finally, Table 5 presents the sodium photon return results obtained at Cerro Pachón during the period of November 2011 through May 2012. $\mathrm{P}_{\text {laser }}$ in the second column is the average GS laser system output power plotted in green in Fig. 3. $\mathrm{P}_{\text {projected }}$ per LGS in the third column is the projected laser power per LGS based on $\mathrm{P}_{\text {laser }}$ and the corresponding LGSF throughput listed in Table 3. Values listed in the fourth column (returns @ LGS WFS per LGS per Watt of projected laser power) are taken directly from Table 1 in ${ }^{18}$.

Results in terms of sodium photon return per LGS per Watt of projected laser power (fifth column) show that the GS LGSF does not meet its per Watt specification during the months of November 2011, December 2011 and January 2012 (corresponding to low sodium season), by a factor of two to three. Results however are within the per Watt specification range during the months of February, April and May 2012 when sodium abundance is increasing and expected to peak around May. Low photon returns obtained in March 2012 either show that we made a mistake in our data reduction process for that month, or they more likely confirm the fact that night-to-night and month-to-month sodium photon return variations are so large and hard to predict that robust AO systems should be designed with significant margins to cope with them.

A factor of two discrepancy between the sodium return per Watt specification and achieved results can partially be explained by what has been learned since 2001 about optimal, and conversely sub-optimal, laser formats ${ }^{19,20,21}$. The large $\sim 2 \mathrm{GHz}$ spectral bandwidth of the GS laser system is partly responsible for this discrepancy. Other, more subtle physical effects involved in the GS laser to mesospheric sodium atom interaction efficiency are also likely at play.

Comparing sodium photon returns per LGS achieved in the November 2011-May 2012 period to the 2001 MCAO PDR specification leads to the disappointing conclusion that the GS LGSF does not meet specification by a factor of two to three for the lower end of the specification range $\left(166 \mathrm{photons} / \mathrm{cm}^{2} / \mathrm{s}\right)$ and by a factor of two to five for the higher end of the specification range $\left(257\right.$ photons $\left./ \mathrm{cm}^{2} / \mathrm{s}\right)$. The factor of two in both cases can readily be explained by the low GeMS system throughput mentioned earlier in this section. Larger discrepancy factors are also partly explained by the undesirably large spectral bandwidth of the GS laser and other physical effects, including of course sodium abundance variations. As explained in ${ }^{7}$, when sodium photon returns fall short of specification, the principal mitigation strategy is for GeMS to be run at lower frame rates than the specified $800 \mathrm{~Hz}$, typically down to frame rates as low as 100 to $200 \mathrm{~Hz}$ in low sodium abundance season.

Table 3: Comparison of LGSF/MCAO bench throughputs which had been assumed at the time of the MCAO Preliminary Design Review in 2001 to derive the GS LGSF photon return requirements per LGS, vs. estimated or measured values in 2011-2012A. Atmospheric transmission is not included.

\begin{tabular}{|l|c|c|c|}
\hline Throughput or QE @ 589nm & 2001 assumptions & $\begin{array}{c}\text { Nov-Dec 2011 estimates } \\
\text { (pre-LLT in-situ wash) }\end{array}$ & $\begin{array}{c}\text { Jan-May 2012 estimates } \\
\text { (post-LLT in-situ wash) }\end{array}$ \\
\hline \hline BTO & 0.8 & 0.55 & 0.55 \\
\hline LLT & 0.9 & 0.88 & 0.95 \\
\hline$L G S F$ & 0.72 & 0.48 & 0.52 \\
\hline \hline GS telescope & 0.8 & 0.8 & 0.8 \\
\hline MCAO bench & 0.7 & 0.6 & 0.6 \\
\hline LGS WFS detector QE & 0.85 & 0.8 & 0.8 \\
\hline Telescope+AO $(Q E$ included) & 0.48 & 0.38 & 0.38 \\
\hline \hline GeMS & $\mathbf{0 . 3 5}$ & $\mathbf{0 . 1 8}$ & $\mathbf{0 . 2 0}$ \\
\hline
\end{tabular}

Table 4: GS LGSF sodium photon return specifications as written in 2001 for the MCAO Preliminary Design Review, in terms of photo-detection events at the LGS WFS as well as in terms of flux at the primary mirror of the GS telescope.

\begin{tabular}{|c|c|c|c|}
\hline $\begin{array}{l}\text { Photon return per } \\
\text { LGS at zenith }\end{array}$ & Specifications & $\begin{array}{c}\text { Specifications per Watt of } \\
\text { projected laser power }\end{array}$ & Comments \\
\hline (a) LGS WFS & $\begin{array}{c}250-390 \mathrm{ph} / \mathrm{subap} / \text { frame } \\
62-97 \mathrm{ph} / \mathrm{pix} / \text { frame } \\
80-125 \mathrm{ph} / \mathrm{cm}^{2} / \mathrm{s}\end{array}$ & $11-17 \mathrm{ph} / \mathrm{cm}^{2} / \mathrm{s} / \mathrm{W}$ & $\begin{array}{c}800 \mathrm{~Hz} \text { frame rate } \\
50 \times 50 \mathrm{~cm}^{2} \text { subapertures } \\
4 \text { pixels per subaperture }\end{array}$ \\
\hline a, M1 & $166-257 \mathrm{ph} / \mathrm{cm}^{2} / \mathrm{s}$ & $23-36 \mathrm{ph} / \mathrm{cm}^{2} / \mathrm{s} / \mathrm{W}$ & \\
\hline
\end{tabular}


Table 5: Range of GeMS sodium photon returns measured per LGS during the 2011B and 2012A commissioning, and comparison to GS LGSF specifications. Photon returns are calculated based on LGSF and Telescope + AO throughputs presented in Table 3.

\begin{tabular}{|c|c|c|c|c|c|}
\hline Month/Year & $\begin{array}{l}\mathbf{P}_{\text {laser }} \\
\text { (W) }\end{array}$ & $\begin{array}{c}P_{\text {projected }} \\
\text { per LGS (W) }\end{array}$ & $\begin{array}{c}\text { Return @ LGS } \\
\text { WFS per LGS per } \\
\text { W of projected } \\
\text { power }\left(\mathrm{ph} / \mathrm{cm}^{2} / \mathrm{s} / \mathrm{W}\right) \\
\end{array}$ & $\begin{array}{c}\text { Return @ M1 } \\
\text { per LGS per W of } \\
\text { projected power } \\
\left(\mathrm{ph} / \mathrm{cm}^{2} / \mathrm{s} / \mathrm{W}\right) \\
\end{array}$ & $\begin{array}{c}\text { Return per LGS @ } \\
\left.\text { M1 (ph/ } / \mathrm{cm}^{2} / \mathbf{s}\right)\end{array}$ \\
\hline $\begin{array}{l}2001 \text { specifications } \\
\text { (low sodium season) }\end{array}$ & 50 & 7.2 & $11-17$ & $23-36$ & $166-257$ \\
\hline Nov 2011 & 41 & 4.0 & 4.8 & 12.5 & 50 \\
\hline Dec 2011 & 38 & 3.7 & 3.5 & 9.1 & 34 \\
\hline Jan 2012 & 30 & 3.1 & 4.1 & 10.7 & 33 \\
\hline Feb 2012 & 31 & 3.2 & 8.2 & 21.4 & 69 \\
\hline Mar 2012 & 38 & 4.0 & 6.5 & 16.9 & 67 \\
\hline Apr 2012 & 38 & 4.0 & 11.5 & 29.9 & 119 \\
\hline May 2012 & 33.5 & 3.5 & 13 & 33.9 & 119 \\
\hline
\end{tabular}

\subsection{LGS Spot Size}

The GS LLT was installed on the GS telescope in late 2007. Results of the LLT image quality tests that were performed shortly thereafter using a Natural Guide Star (NGS) are reported in ${ }^{4}$. Based on those results, we predicted that the LLT should be capable of creating an intrinsic LGS spot size (with the contributions of uplink seeing and spot elongation removed) on the order of 1.0 arcsecond on the sky, consistent with LGS spot sizes observed at the Gemini North telescope in best seeing conditions. The smallest spot sizes obtained at Gemini North when imaging the LGS with the Gemini acquisition camera over the full $8 \mathrm{~m}$ diameter aperture of the telescope in $\sim 0.6 \operatorname{arcsec}$ seeing are on the order of 1.3 arcseconds Full Width at Half Maximum (FWHM).

LGS spot size results when we first propagated the laser in January 2011 were, as should have been expected, significantly larger than specified since the LLT focus had not been optimized at 589nm yet. Our very first measurement in January 2011 provided an alarming 7 arcsecond LGS spot size at FWHM. LLT focus runs performed during subsequent runs in February, March and April 2011 eventually made it possible to bring this value down to the expected 1.3 arcsecond at FWHM, as shown in Fig. 9 and Fig. 10 below.

\section{LGS spot size (arcsec FWHM) 2011A GeMS commissioning}

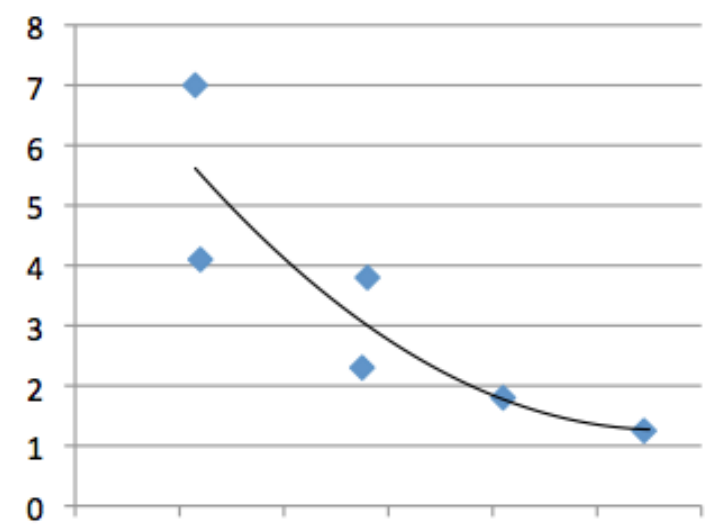

30-Dec 19-Jan 8-Feb 28-Feb 20-Mar 9-Apr 29-Apr

Figure 9: Evolution of LGS spot size during the first few months of the GS LGSF commissioning in 2011A

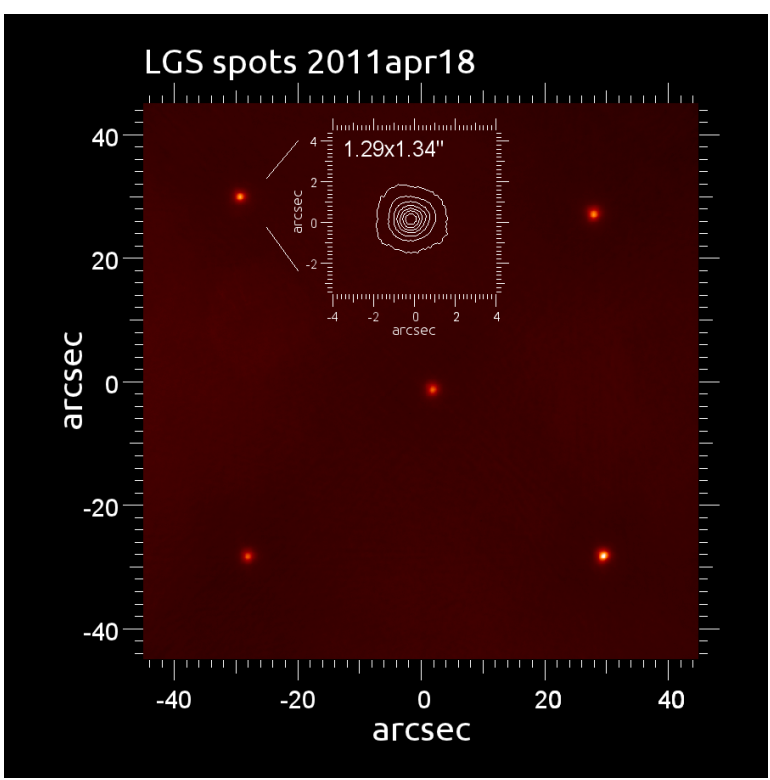

Figure 10: Example of small LGS spot sizes achieved since first light (April 2011 data) 
Since then, LGS spot sizes obtained during the 2011B and 2012A laser runs have ranged between 1.2 (best) and 1.9 (worst) arcseconds FWHM depending on seeing and actual focus optimization, with a distribution centered near 1.7 arcseconds FWHM for the five LGS spots. It is important to note at this point that spot size variations have been observed between the five LGS in the GeMS constellation, on the order of 0.1 to 0.2 arcseconds, typically appearing larger at lower telescope elevations. This effect was attributed to sub-optimal alignment of the five beams on the LLT primary mirror, when each beam would sample a different portion of the LLT pupil aberrations mainly caused by the OAP mounting issues described in ${ }^{4}$.

In November 2011 and March 2012 respectively, a series of approximately two hundred 5ms exposures were taken with the Gemini acquisition camera in order to estimate the intrinsic laser spot size at the sodium layer. Spot size results obtained by lucky imaging to remove the atmospheric contribution were on the order of 1.1 and 1.05 arcseconds at FWHM respectively, thus confirming our earlier estimate of $\sim 1.0$ arcsecond at FWHM based on NGS imaging.

\subsection{LGS Pointing}

The 2001 MCAO PDR pointing requirements for the GS LGSF were three-fold:

(1) Each LGS must be aligned on its respective LGS WFS within 1 arcsecond peak-to-peak accuracy;

(2) The LGS constellation must be positioned on-sky within 1 arcsecond peak-to-peak of the MCAO bench LGS optical axis (the so-called LGS WFS hot spot); and

(3) Each LGS must be stabilized on its respective LGS WFS within 0.05 arcsecond rms at the MCAO sampling rate (maximum of $800 \mathrm{~Hz}$ ).

Section 3 explained how the LGS constellation alignment is ultimately performed on-sky in order to meet specification (1). Following a recent BTOOB re-alignment to improve performance of the BTO PM/CM LUT vs KM angle, the LGSF is now also close to meeting requirement (2): laser operators typically achieve blind pointing accuracies on the order of 2 arcseconds, close enough to specification to enable automated acquisition by the LGS WFS. Finally, closed loop performance of fast jitter compensation by the FSA platforms is illustrated in Fig. 11 below. Tip/tilt residuals are on the order of 100 milli-arcseconds rms, upward of the 50 milli-arcseconds rms specified in (3), but good enough to ensure acceptable GeMS performance. More details on the fast jitter compensation loop can be found in ${ }^{7}$.
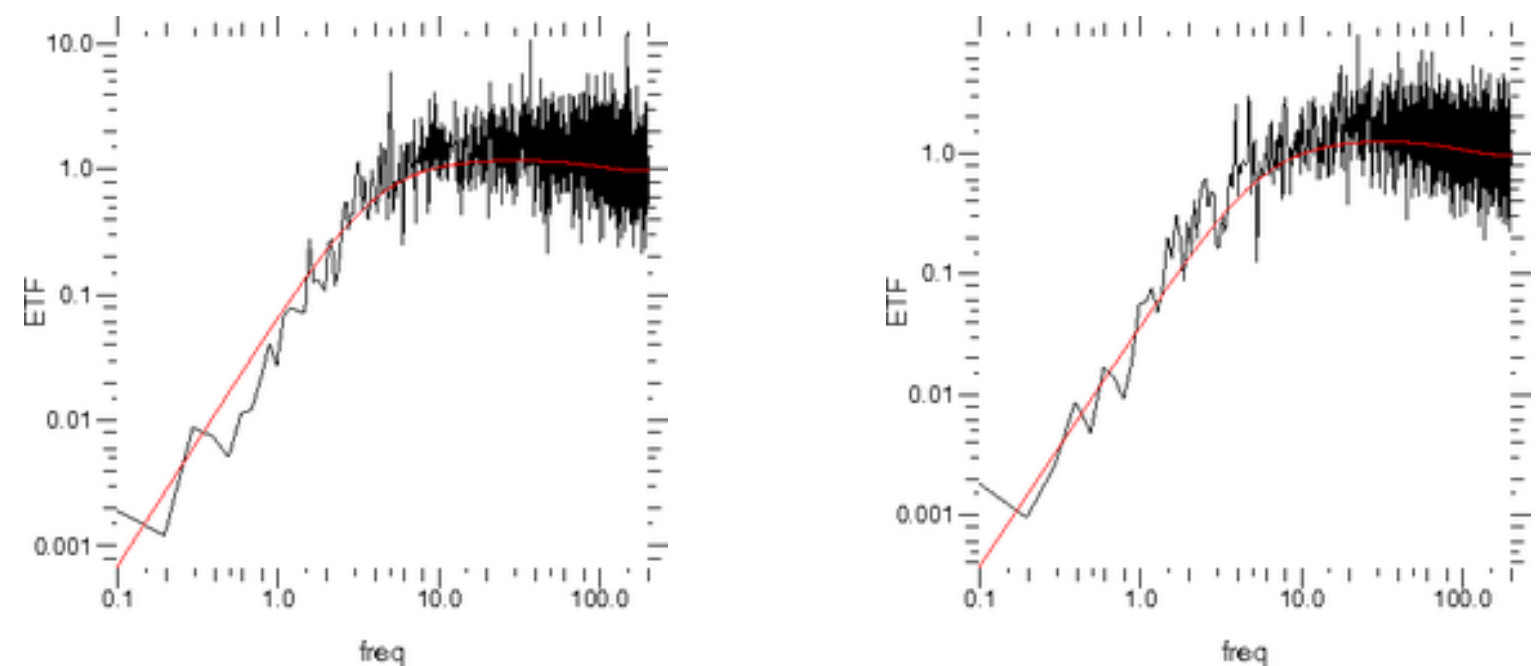

Figure 11: Error Transfer Functions (ETF) for one of the FSA platforms with the loop closed at 200Hz. Expected bandwidths are 10 to 20 times lower than the sampling rate, on the order of 10 to $20 \mathrm{~Hz}$, consistent with these graphs. Left: ETF for Tip; Right: ETF for Tilt. 


\section{LASER GUIDE STAR FACILITY OPERATIONS}

\subsection{Staffing}

Four laser operators were trained and certified during the 2011A, 2011B and 2012A GeMS commissioning runs. Training involved learning all the LGSF operation GUIs, including screens necessary to: operate the GS laser and maintain its optimal performance; align the laser beam(s) though the BTOOP and BTOOB; monitor and adjust laser alignment on the LLT to optimize and balance LGS intensities on the sky; align the LGS constellation on the GeMS LGS WFS; and close the fast tip/tilt laser control loops. These laser operators were experienced laser/optical engineers and technicians who could rapidly adapt to ever changing commissioning conditions, troubleshoot hardware failures as well as contribute to fixing software bugs and implementing software upgrades on-the-fly. Generally speaking, the laser operators have been working closely with the telescope operator, AO scientists and other support engineers to maintain performance records and troubleshooting logs. Their feedback resulted in improved performance and simplified user interfaces, all of which will eventually ease up the transition to normal operations.

A dedicated laser operator will be hired and trained for normal operations. This position will be fully operational beginning October 2012 through the next several years while more improvements are made to enable remote operations from the La Serena Base Facility and eventual handoff for operations by the Gemini Science Operations Specialists (SOS).

One of the most rewarding GeMS operational improvements came from establishing formal laser operator/telescope operator/spotter/AO scientist communication protocols. The laser operator is responsible for pausing the laser propagation sequence during expected and unexpected laser shutter events, resuming laser operations, and if necessary, stabilizing the system as rapidly as possible to minimize the time from laser shuttering to on-sky operational status. The ultimate goal is to reduce the time between non-propagation to full power, on-sky propagation with minimal interruption to observing scientists, and to maximize science acquisition time. Since the formal protocol was introduced and practiced in multiple sessions, we have been able to reduce this time from many minutes to only a few seconds. Currently the longest acquisition sequence corresponds to telescope slews to a new target, when the laser operator must "stabilize" the BTOOP mirror alignment based on predefined Look Up Tables (LUTs) and then manually realign the five laser beams on the LLT. However, thanks to recent software improvements in the way BTO LUT coefficients are handled, this time has been reduced to only a few minutes from the start of telescope elevation slew to full power, on-sky propagation.

\subsection{LGSF Reliability}

LGSF up-time during the most recent commissioning runs was improved to close to $100 \%$ barring other instrument or software glitches that prevented laser propagation as new instruments were being commissioned. The laser maintained a steady output power near $40 \mathrm{~W}$ and a M-squared under the specified 1.4. Wavelength lock was stable throughout each run. Although we do see a small degradation of power and beam quality toward the end of each run, we attribute this effect to small misalignments caused by telescope movement since the laser enclosure is attached to the side of the telescope and by temperature hysteresis. During this 2012 winter shutdown, the laser will be completely realigned which should increase the power output and stability to the specified $50 \mathrm{~W}$. Further laser upgrades will reduce the recovery time to nominal operating conditions.

LGSF operations and reliability is reaching a maturity that even a few months ago seemed beyond expectation. Outstanding performance issues have been identified and are being rectified. We know we need more laser power for better photon return, we know we need to continue to simplify the LGSF user interfaces, we have identified beam transfer issues that need resolution with better constellation alignment through the BTOOP and BTOOB, and we have identified many of the weak links in the LGSF and GeMS LGS WFS mechanical systems. Most issues highlighted or discovered during the 2011-2012A commissioning runs will be corrected during the 2012 GeMS winter shutdown and we are on schedule to resume full operation in November 2012 when the LGSF transfers to normal operations. We are currently updating documentation and preparing for training operational personnel. Our long-term goal of remotely operating the laser from the La Serena Base Facility is closer to becoming a reality. 


\section{CONCLUSION}

The Gemini South Laser Guide Star facility has come a long way since the 2001 MCAO PDR. It required nearly a decade for the LGSF-side of the GeMS project to move through its design, fabrication, integration and test phases. Progress was largely paced by the challenging procurement of a state-of-the-art $50 \mathrm{~W}$ laser system for GeMS. However when the GS laser finally arrived on Cerro Pachon, it took less than a year for all AO and LGSF subsystems to come together and for the GeMS team to achieve laser first light on January 22, 2011. It was the first time in all LGS AO history that a sodium LGS constellation was projected on-sky, ready to be used for MCAO observations at an 8mtelescope observatory.

Commissioning of the Gemini MCAO system and its Laser Guide Star facility has been on going on a monthly basis throughout 2011 and 2012A, only interrupted by the June-October 2011 GeMS winter shutdown. During its 2012 winter shutdown, GeMS will be getting ready to move into science verification when laser operation resume in November 2012. The system is expected to be offered for normal science observations in 2013.

Multiple upgrades have already been implemented to improve the LGSF performance and its reliability. Although sodium photon returns are not nearly as high as desired to enable maximum MCAO corrections at all times, mitigation strategies will still make it possible to operate year-round, including during the low sodium season in summer. The GeMS laser team is now actively preparing the LGSF transition to normal operations by a dedicated laser operator starting in November 2012.

\section{ACKNOWLEGMENTS}

The Gemini Observatory is operated by the Association of Universities for Research in Astronomy, Inc., under a cooperative agreement with the NSF on behalf of the Gemini partnership: the National Science Foundation (United States), the Science and Technology Facilities Council (United Kingdom), the National Research Council (Canada), CONICYT (Chile), the Australian Research Council (Australia), Ministério da Ciência e Tecnologia (Brazil) and Ministerio de Ciencia, Tecnología e Innovación Productiva (Argentina).

\section{REFERENCES}

[1] Ellerbroek, B. L., Rigaut, F. J., Bauman, B. J., et al., "MCAO for Gemini South," Proc. SPIE 4839, 55-66 (2003).

[2] D'Orgeville, C., Bauman, B. J., Catone, J. W., et al., "Gemini north and south laser guide star systems requirements and preliminary designs," Proc. SPIE 4494, 302-316 (2002).

[3] Boccas, M., Rigaut, F., Gratadour, D., et al., "GeMS: Gemini Mcao System: current status and commissioning plans," Proc. SPIE 7015, 70150X (2008).

[4] D'Orgeville, C., Daruich, F., Arriagada, G., et al., "The Gemini South MCAO laser guide star facility: getting ready for first light," Proc. SPIE 7015, 70152P (2008).

[5] Neichel, B., Rigaut, F., Bec, M., et al., "The Gemini MCAO System GeMS: nearing the end of a lab-story," Proc. SPIE 7736, 773606 (2010).

[6] D’Orgeville, C., “Laser first light at Gemini South”, Gemini Focus (June 2011)

[7] Neichel, B., Rigaut F., Arriagada G., et al., "Status and performance characterization of the Gemini MCAO systems," Proc. SPIE 8447, 8447-32 (2012).

[8] Rigaut, F., Neichel, B., Boccas, M., et al., "GeMS: first on-sky results," Proc. SPIE 8447, 8447-18 (2012). 
[9] Hankla, A. K., Bartholomew, J., Groff, K., et al., "20-W and 50-W solid-state sodium beacon guidestar laser systems for the Keck I and Gemini South telescopes," Proc. SPIE 6272, 62721G (2006).

[10] Lee, I., Jalali, M., Vanasse, N., et al., "20 W and $50 \mathrm{~W}$ guidestar laser system update for the Keck I and Gemini South telescopes," Proc. SPIE 7015, 70150N (2008).

[11] Sawruk, N., Lee, I., Jalali, M., et al., "System overview of $30 \mathrm{~W}$ and $55 \mathrm{~W}$ sodium guide star laser systems," Proc. SPIE 7736, $77361 Y$ (2010).

[12] Pentland, G. J., Blanco, D., Rebeske, K., et al., "Preliminary design of a laser launch telescope for Gemini," Proc. SPIE 4839, 429 (2003).

[13] Telle, J., Drummond, J., Denman, C., et al., "Studies of a mesospheric sodium guidestar pumped by continuouswave sum-frequency mixing of two Nd:YAG laser lines in lithium triborate," Proc. SPIE 6215, 62150K (2006).

[14] ANSI Z136.1-2007: “American Standards for Safe Use of Lasers” (2007).

[15] ANSI Z136.6-2005: “American National Standard for Safe Use of Lasers Outdoors” (2005).

[16] FAA Order 7400.2D Ch.34: Outdoor Laser / High Intensity Light Demonstrations.

[17] Summers, D., Gregory, B., Stomski, P. J., et. al, "Implementation of a Laser Traffic Control System supporting Laser Guide Star Adaptive Optics on Mauna Kea," Proc. SPIE 4839, 440 (2002).

[18] Neichel, B., Callingham, J., D’Orgeville, C., et al., "Characterization of the sodium layer at Cerro Pachon and impact for GeMS performance," Proc. SPIE 8447, 8447-176 (2012).

[19] Moussaoui, N., Holzlohner, R., Hackenberg, W., and Calia, D. B., "Dependence of sodium laser guide star photon return on the geomagnetic field," A\&A 501, 793-799 (2009).

[20] Holzlohner, R., Rochester, S., Calia, D. B., and Budker, D., "Optimization of CW sodium laser guide star efficiency," A\&A 510, A20 (2010).

[21] Rochester, S., Otarola, A., Boyer, C., and Budker, D., "Modeling of pulsed laser guide stars for the thirty meter telescope project," arXiv:1203.5900 (2012). 